\title{
Model-based mechanical design of a passive lower-limb exoskeleton for assisting workers in shotcrete projection
}

\author{
Stefano L. Capitani • Matteo Bianchi • Nicola Secciani (i) - Marco Pagliai • \\ Enrico Meli · Alessandro Ridolfi
}

Received: 27 April 2020/Accepted: 28 November 2020/Published online: 12 December 2020

(C) The Author(s) 2020

\begin{abstract}
Despite the current industrial trend towards automation, many workers are still daily exposed to heavy loads during their duties. Regarding the construction industry, the shotcrete projection results in a particularly arduous whole-body effort for the so-called "concrete reinforcement workers". In this paper, the design of a passive robotic human assistance tool to assist workers in dealing with the specific task of shotcrete projection will be described. If the design of an effective aid plays a crucial role in improving the job's quality, following the guidelines given by the French company Eiffage Infrastructures; besides, the acceptance of the proposed solution shall have special consideration. Therefore, starting from an in-site assessment of the scenario, the presented solution has been profoundly adjusted to address the issues coming from this particular task. The exoskeleton adaptability to different lower-limb activities has been preserved without lowering its comfort during daily life in the working area. The developed solution's operation range has been specifically optimized to assist the worker during specific efforts without hindering the other movements.
\end{abstract}

S. L. Capitani · M. Bianchi · N. Secciani ( $)$.

M. Pagliai · E. Meli · A. Ridolfi

Department of Industrial Engineering, University of Florence, Via di Santa Marta, 3, 50139 Florence, Italy

e-mail: nicola.secciani@unifi.it
Keywords Wearable robotics - Exoskeletons for workers · Passive lower-limb exoskeletons · Taskoriented design

\section{Introduction}

The increasing need for flexibility in the industry claims more and more workers on the shop floor, especially in highly-automated environments. Indeed, some production processes can not be fully automated due to frequent changes of activity, product type, or order size. In all of these cases, human intervention is necessary, and the prevention of manual handling injuries is intensely required. Despite the on-going efforts in reducing the physical load and the risk of injury, many workers are still exposed to physical workloads due to material handling (over $30 \%$ of the work population in the EU), repetitive movements (63\%), and awkward body postures (46\%) [1]. A statistical analysis dated back to the early $2000 \mathrm{~s}$ showed that three-quarters of the involved construction workers experienced at least one musculoskeletal disorder per year [2]. The same study also highlighted pain in the lower back as the most frequent symptom. In recent years, another survey has confirmed the same disorders at the top of the causes of absence from work [3]. Finally, data from the 2018 "Injuries, Illnesses, and Fatalities" database of the Bureau of Labor Statistics (BLS) shows that, among more than 200 
thousand episodes in the United States alone, still the $40 \%$ of the total injuries saw the back involved.

In response to this ongoing problem, the exploitation of wearable robotic devices, such as exoskeletons, might be a practical approach to reduce the inherently involved health risk [4-8]. At the same time, such a solution will fully profit from human creativity and flexibility, which still cannot be demanded to any robot systems (e.g., classical robots, full-automation systems, etc.). Following the reasonings above, the presented work deals with the design of an assistive lower-limb exoskeleton for construction workers (Fig. 1).

The idea arose to tackle a specific need of Eiffage Infrastructures, a French civil engineering construction company: assisting the workers during the shotcrete projection. This particular task requires the worker to deal with heavy objects in rough locations, sometimes, hardly reachable by the worker himself. Thus, fully automated solutions (e.g., ground robots or automatic cranes, already in use by the company) are not an option suitable for the case under examination. Therefore, a tailor-made solution shall be provided to prevent accidents at work in the present and occupational diseases in the future. The shotcrete projection also forces the body to an unnatural posture that dangerously strains the lumbar part; this may damage the back and evolve into occupational diseases.

According to the nature of the job involved, assisting the workers in weight-lifting is the second key characteristic of the proposed exoskeleton. As well known, the incorrect performing of such a task has high chances to lead to back injuries. Finally, since the proposed device is specifically designed to assist the construction workers, it will also have to be transparent ${ }^{1}$ to the "building site life" (i.e., all the secondary activities, as walking or stair climbing/ descending, that a construction worker may perform during his duties).

Consequently, the exoskeleton's primary aim is to assist the workers with loads and prevent them from assuming incorrect postures during the shotcrete

\footnotetext{
${ }^{1}$ Transparency, for an exoskeleton, is meant as the ability to perform all the assigned tasks without creating discomfort or perception issues for the user. In this context, transparency can thus be intended as the characteristic of the device not to influence the activities of the wearer, who should not feel, as much as possible, to wear it.
}

projection and weight-lifting. In parallel, other three aspects have been identified as side requirements: (1) transparency during walking; (2) sufficient freedom to climb a step; (3) high wearability, since the exoskeleton shall be worn for many hours and shall not cause any issues as rubbing, excessive pressure on the tissues, or parasitic joint loads.

\subsection{Main contribution}

The prevention of occupational diseases is taking the form, in recent years, of a race for workers' technological equipment. Companies, when possible, try to replace the man with a machine in extremely heavy tasks. Nevertheless, when this is not a viable path, it is preferable to provide the worker with an exoskeleton that reduces effort and fatigue. In the construction industry, the shotcrete projection results to be significantly spread as a practice and arduous in terms of whole-body effort, and, besides, it is very likely not to go out of fashion in the next years. This task may not always be carried out with the exclusive use of machinery due to the wide variety of working conditions (e.g., confined spaces, unstable terrain). Moreover, most of the other available solutions are not entirely compliant with this specific job since all of them stem from a deep specific-task-oriented design procedure, which is mandatory for the development of a useful aid tool (see Sect. 2 for further details). Following the reasonings above, the main contribution of the research work presented in this paper is the development of a model-based design strategy leading to the embodiment of a lower limb passive support system for assisting concrete reinforcement workers. Finally, if the possibility of designing a worker aid adaptable to general tasks could be convenient from the industrial point of view, a more specific solution might be much more effective from the worker's point of view. The proposed solution has been designed to tackle the issues coming from the shotcrete projection task mainly and adapt to be worn throughout the work shift.

\subsection{Paper structure}

The paper is organized as follows. Section 2 describes some of the main solutions commercially available. Section 3 introduces the issue addressed in this work. Section 4 reports a detailed description of the adopted 


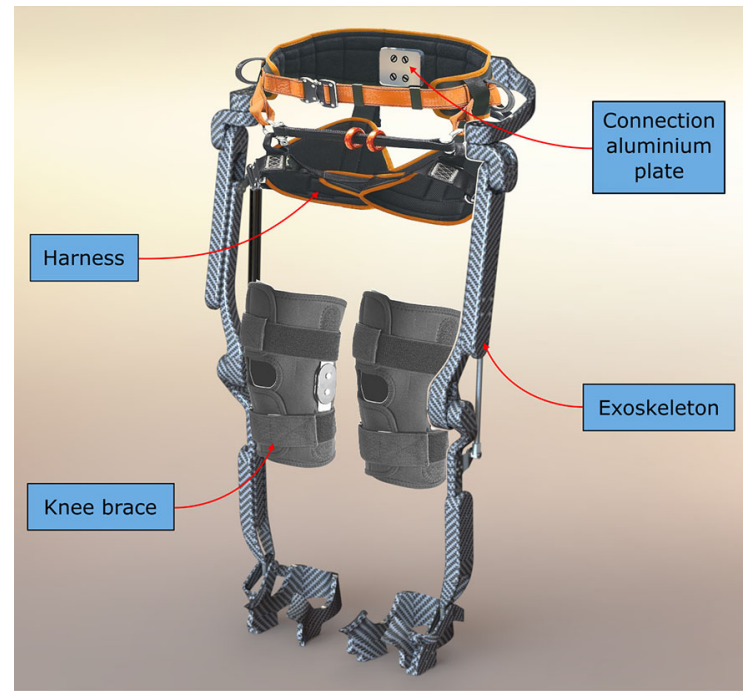

Fig. 1 The proposed overall concept for the assistive passive lower-limb exoskeleton for construction workers

worker's dynamic model. Section 5 described the kinematic and dynamic model exploited to design the proposed exoskeleton. Section 6 reports the evaluation of the assistance level to the leg joints. Section 7 discusses the main points of the work and concludes the paper.

\section{Exoskeletons in the industry}

Many are the available commercial products and research works that embody effective solutions to assist workers during their daily tasks. The birth of a remarkable number of different exoskeletons can be due to two principal reasons. On the one hand, this can be ascribed to a rising need for such solutions in the industry. On the other hand, these devices are often designed to perform particular tasks; therefore, defining a solution compliant with different jobs can be quite a challenge. Consequently, the number of exoskeletons varies according to the number of different tasks. Many companies have hence started developing their own type of exoskeleton, precisely thought to help workers executing their ordinary tasks on their specific shop floor.

Below some of the most advanced and recent examples of lower-limb or back-support exoskeletons are reported. These 2 categories of devices have been selected because they were more suited to the type of work for which the commissioning company, Eiffage Infrastructures, required the exoskeleton's development.

\subsection{HAL-LB03 by cyberdyne}

This device is a commercial solution designed to prevent the onset of back pain in workers dealing with the lifting of bulky goods. It is a lightweight and active lumbar module that anchors to the lower back and thighs to provide torque-assistance to the hip joint.

\subsection{Chairless chair 2.0 by nonee}

As the name suggests, this particular exoskeleton offers the user the possibility of sitting on it. It aims to prevent back pain, providing a seat to be used during prolonged desk tasks. It is a passive lower-limb device that anchors to the lower back, thighs, and shoes.

\subsection{Archelis by nitto}

This lower-limb exoskeleton is designed to assist surgeons during their shifts in the operating room. During any interventions, surgeons have to change position many times to access the various equipment. Since surgery can take up to several hours, this device is designed to provide support to reduce leg and back fatigue. Archelis is passive and has three connection points for each leg: on the shoe, shank, thigh.

\subsection{Model Y and HIMICO by atoun}

Model Y takes its name from the shape it draws on the user's back, from the neck to the hips. Similar to HALLB03, it is designed to assist with heavy weight lifting actively, but to the two anchor points of the previous one, it adds a harness for the shoulders. It is claimed that it can also boost the gait and stair climbing. HIMICO is instead still at a prototypical level and focuses on gait assistance. It is composed of a case that houses batteries and motors, placed on the lower back, and some harnesses for moving the legs.

\subsection{Discussion}

Each of the presented products has been developed for a particular task. Chairless Chair 2.0 and Archelis are entirely passive. This means that the level of 
assistance they offer comes from the system's mechanical configuration and does not use any external energy source. HAL-LB03, Model Y, and HIMICO use instead a battery's electrical energy to assist the wearer through electric motors. Besides, Chairless Chair 2.0 and Archelis have a structure that has the advantage of unloading part of its weight on the ground. HAL-LB03, Model Y, and HIMICO are smaller but do not provide the same benefit. Finally, although very similar, the devices within these 2 groups present features that differentiate them, e.g., the posture, the assistance level, or the control strategy.

The exoskeleton, whose design process is now presented, takes its cue from these solutions. However, it will also need to be deeply adapted to assist workers effectively during shotcrete projection and be compliant with the requirements from Eiffage Infrastructures. Indeed, these commercial products are not thought to undergo the construction site life and are mainly designed to assist with vertical forces, namely the bodies' gravity. Shotcrete projection instead involves heavy loads also in the horizontal direction.

This new exoskeleton's design will be inspired by Chairless Chair 2.0 and Archelis in terms of structure and passive assistance. Walking several kilometers a day through the construction site, workers need a device that does not fatigue them with excessive weight. When it comes to reducing the weight of a device, a passive solution is usually preferable over an active one for the absence of power supply. Besides, the lack of electric parts means improved safety, especially when surrounded by a harsh environment as the construction site is. However, because of the loads from the shotcrete projection, this exoskeleton shall also embed support for the lumbar area similarly to HAL-LB03, Model Y, and HIMICO. Not to mention that assisting in lifting heavy loads is also an interesting option for this device.

Therefore, the solution presented here tries to summarize the different characteristics of the stateof-the-art exoskeletons that make it more suitable for this particular application.

\section{Shotcrete projection task}

When it comes to designing an assistive wearable system, knowing what the body faces in terms of interactions with the external environment is mandatory. In this case, such interactions result in the loads exchanged between the worker and the hose during shotcrete projection (Fig. 2).

The involved forces can be split into three main contributions: (1) the total weight of the hose and the flowing concrete; (2) the elastic force of the hose when the worker forces it to assume the bent pose required to perform the task; (3) the fluid-dynamic backlash, which is caused by the projection of pressured concrete through the hose nozzle. Both the concrete flow and the worker's gestures (those required to spread the concrete over the wall homogeneously) have a specific frequency that has to be considered. Therefore, the resulting loads have been examined both in terms of frequency and magnitude. As visible in Fig. 2, workers usually hold the hose between their knees during the projection, they do this to gain control of the hose motion and to unload part of the load on the ground through the legs. For this reason and wanting to focus primarily on back fatigue, the only portion of hose considered in this analysis will be the one that from the point of narrow flexion between the knees ends with the nozzle.

\subsection{Load frequency}

A measurement campaign has collected frequency data during an on-site inspection, expressly carried out to identify the load frequency that burdens the worker's body. These measurements have been gathered through the MTi28A53G35 Inertial Measurement Unit (IMU) by Xsens (Enschede, Netherlands). The sensor has been mounted as close as possible to the

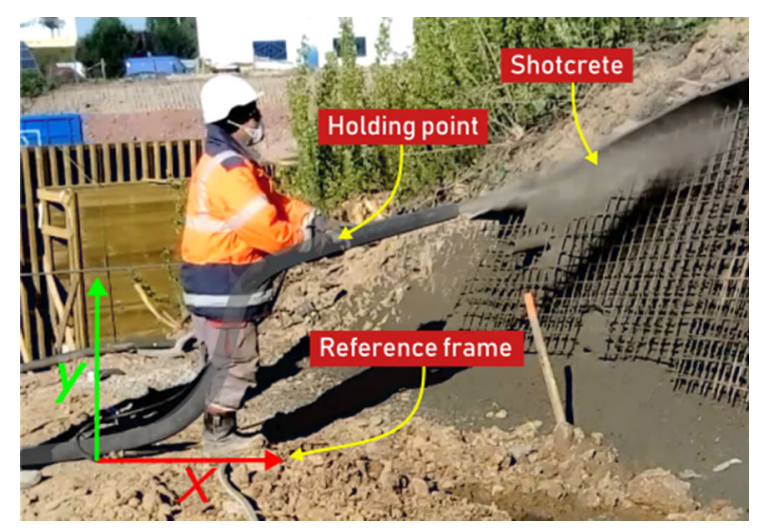

Fig. 2 A typical pose for the worker while holding the hose during a vertical-wall shotcrete projection 


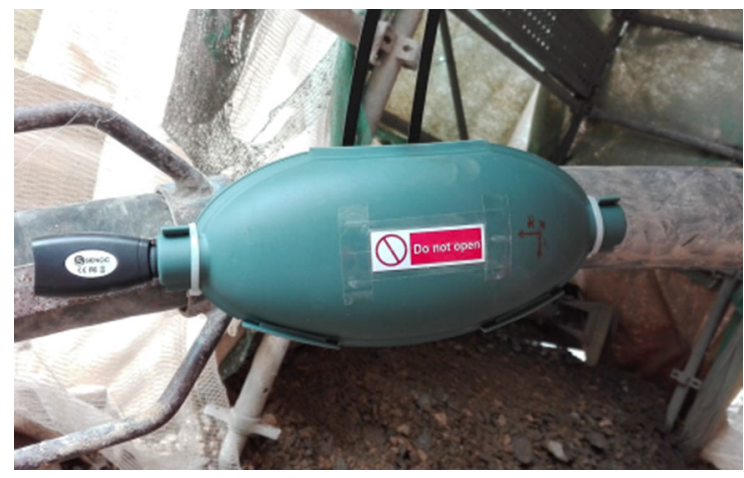

Fig. 3 The measurement setup exploited to measure the frequency of the interaction force

interaction point between the hose and the worker; namely, the iron handles visible in Fig. 3. Two workers have been involved in the study. Each worker has been asked to work as usual, and seven measurement sessions have been recorded for each worker for a total of 14 different recorded sessions.

The assessed frequencies have been studied in the three principal directions, which, referring to the body anatomy and the reference frame shown in Fig. 2, are the craniocaudal (y-axis), dorsoventral (x-axis), and leftright (z-axis) directions. The spectral analysis performed on the collected signals - by exploiting a standard Fast Fourier Transform (FFT) algorithm - has highlighted a load frequency of about $2.75 \mathrm{~Hz}$ along the craniocaudal and left-right axes; no relevant frequency along the dorsoventral axis. An average oscillation of $\pm 10^{\circ}$ has been recorded both around y-axis and $\mathrm{z}$-axis.

\subsection{Load magnitude}

As anticipated, the total interaction force the worker and the hose exchange during the concrete projection consists of three main components. Fig. 4 will be the reference for all the calculations presented in this subsection.

\subsubsection{Weight component}

The first component is the one resulting from the sum of the hose's weight and the weight of the concrete flowing through it, taking into account the whole part of hose lifted off the ground by the worker ${ }^{2}$. The mass

\footnotetext{
2 The on-site experience has led the authors to consider the one of Fig. 2 as the worst-case scenario in terms of hose length lifted from the ground
}

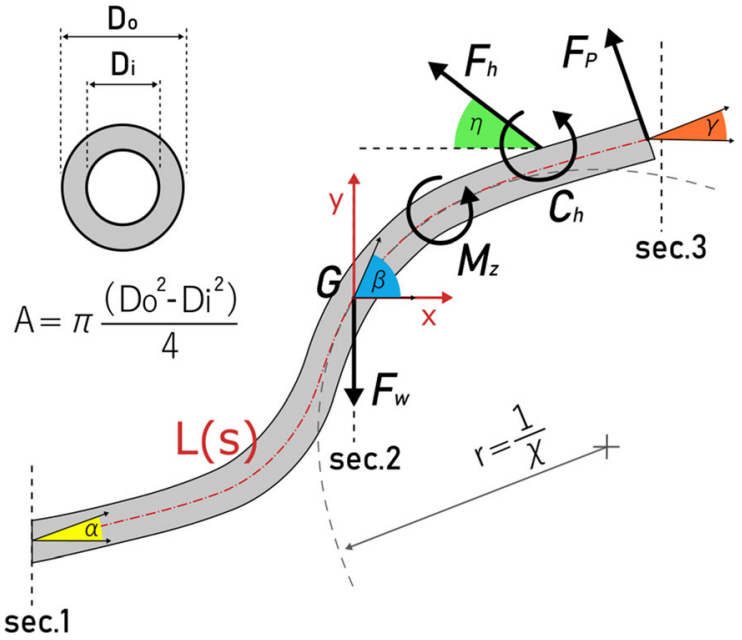

Fig. 4 The overall scheme of the hose load and geometric configuration used as reference for the dynamics calculation. Section 1 is the point where the hose is lifted from the ground, sect. 2 refers to the barycentric section of the hose, sect. 3 is the nozzle outlet

of the concrete, $m_{c}$, has been calculated by geometry-knowing its density—while a portion of the hose-provided by the company-has instead been weighed on a scale, and then its mass per meter, $m_{h}$, has been calculated knowing its length. The contribution to the whole load could thus be calculated as follows:

$F_{w}=g\left(m_{h} l_{h}+m_{c}\right)$,

$M_{w}=F_{w}\left(x_{h}-x_{g}\right)$.

Where $F_{w}$ and $M_{w}$ are, respectively, the resultant force and momentum of the weight force, $l_{h}$ is the length of the lifted portion of the hose calculated along its streamline $L(s)$ between sects. 1 and $3, x_{h}$ is the abscissa of the holding point, and $x_{g}$ is the abscissa of the center of gravity of the hose and the concrete, $G$.

\subsubsection{Elastic component}

This component stem from the hose elasticity, which, when the hose is forced to assume the shape shown in Fig. 2, tends to make it straighten out again. Considering the Euler-Bernoulli beam theory, the relationship between the bending torque $M_{z}$ about $z$-axis and the elastic curvature $\chi$ is given by: 
$M_{z}=k_{\chi} \chi$,

where $k_{\chi}=E / J$ is the bending stiffness ( $E$ is the Young's modulus of the material, available from the hose datasheet, $J$ is the moment of inertia of the section), and $\chi$ is the bending curvature, defined as the inverse of the bending radius $r$ :

$\chi=\frac{1}{r}$.

\subsubsection{Fluid-dynamic component}

Referring to Fig. 4 and aiming at the identification of the effect of the concrete flow, the force that now will be investigated is $F_{P}$. The momentum balance equation has been applied to each $i$-th section to solve the problem:

$\sum_{A} F_{i}=\frac{\partial}{\partial t} \int_{V} \rho v d V+\int_{A}(\rho v) v d A$,

where $\rho$ is the concrete density, $v$ is the flow speed, $A$ is the section area and $V$ is the concrete volume. The left term of Eq. 5 represents the resultant fluid-dynamic force acting on the hose. The first term on the right is the variation of the momentum within the volume and is null in steady state. The second term on the right is instead the flow variation of the momentum between inlet and outlet.

The force resulting from the resolution of Eq. 5 (namely the forces on the hose portion between sects. 1 and 3) can thus be defined by 10 parameters:

$$
F_{P}(\gamma, t)=f\left(p_{3}, A_{3}, v_{3}, \gamma(t), p_{1}, A_{1}, v_{1}, \alpha, \rho\right),
$$

where $p_{i}, A_{i}, v_{i}, \alpha$ and $\gamma$ are, respectively, the pressure, the section area, the flow speed, and the the angles between the horizontal and the hose streamline $L(s)$ at sects. 1 and 3. The flow density ${ }^{3}(\rho)$ has been assumed constant, while the flow outlet angle $\gamma(t)$ has been made varying with a sinusoidal trend in accordance to the results of Subsect. 3.1.

The geometric quantities, i.e., $A_{2}, A_{3}$ and $\beta$, have been assumed known by considering a constant hose section and a fixed configuration of the hose. For the sake of simplicity, while the loads have been

\footnotetext{
${ }^{3}$ Density of the concrete mix: average value of the pressures considered between the sections.
}

considered as a function of the time, the hose configuration has been instead treated as time-invariant. The general flow speed $v_{i}$ has been calculated by knowing the concrete volume $V$ ejected in the time interval $T$, through a hose section of inner diameter $D_{i}$ :

$$
v_{i}=\frac{V}{T \pi\left(\frac{D_{i}}{2}\right)^{2}} .
$$

Finally, under the simplifying hypothesis that the system operates in steady state, all the other parameters can be considered time-invariant, and the Bernoulli's principle for non-compressible flows has been used to identify the pressure parameters:

$p_{1}=\rho R+p_{3}$,

where $p_{3}$, which is the pressure at the outlet of the hose (sec.3), has been considered equal to the atmospheric pressure, and $R$ is the parameter representing all the pressure losses inside the hose.

\subsection{Interaction between the hose and the worker}

In the previous subsections, all the interaction forces between the worker and the hose have been detailed. Moving on to the final study of this interaction, it is necessary to start with two assumptions. The first one implies that the point of contact between workers and hose remains fixed. This hypothesis appears reasonable in the light of the fact that, as visible in Fig. 3, the tube presents some handles. As the second assumption, it has been decided to neglect all the non-vertical forces and all the torques along the $\mathrm{x}$-axis and $\mathrm{y}$-axis. Also, this hypothesis seems logical since of the three types of load (weight, elastic, and fluid-dynamic) only the last one has small quantities outside those considered.

The resultant loads reported at the holding point, namely the force $F_{h}$ and the momentum $M_{h}$, can be therefore defined by the following vector equations:

$$
\begin{aligned}
& F_{h}(\gamma, t)=F_{P}(\gamma, t)+F_{w}, \\
& M_{h}(\gamma, t)=M_{w}+M_{z}+M_{P}(\gamma, t),
\end{aligned}
$$

where $M_{P}$ is the momentum generated when moving the point of application of $F_{P}$ to the holding point. The 
loads reported above, however, are stationary, while the frequency contribution, introduced in Subsect. 3.1, has still to be considered. The outlet angle $\gamma$ has been considered as a time-variant parameter varying in a range between $-10^{\circ}$ and $10^{\circ}$ concerning the horizontal line with a pulse frequency of $2.75 \mathrm{~Hz}$. Therefore, the calculated loads were all solved as functions of $\gamma$ by implementing the corresponding dynamic model in MATLAB.

\section{Worker's dynamic model}

Once the external forces acting on the worker has been identified, a multibody dynamic model has been implemented within Simscape ${ }^{\mathrm{TM}}$ environment to define their effects on the worker's body. The human body dynamic model has been built upon the information available in literature [9]. The parameters used to define each body segment weight have been taken from the literature as well as [10-12]. While doing so, a male subject, $1755 \mathrm{~mm}$ height and $78.4 \mathrm{~kg}$ weight, has been considered for a first tentative. These data refer to a male 50th percentile and are also compliant with those reported in S.R. CEN ISO/TR 7520-2:2011, the currently used standard. Any small or large differences in weight and height of the different workers will be investigated later to realize different sizes of the same device. Besides, the design of specific adjustment systems will make the exoskeleton easily adaptable to different body types.

If well characterized, this model would have allowed not only to evaluate how the shotcrete projection affects the wearer and assess the transparency and effectiveness of the designed exoskeleton. A parametric model, which exploits just the weight and height of a subject has been finally developed.

\subsection{Model validation}

The parametric model obtained from the literature has been validated via the Motion Capture (MoCap) technique. Because of the impossibility of validating the shotcrete projection itself (it has not been possible to mock that gesture within a MoCap studio on the working site), the authors exploited the MoCap to assess the goodness of the dynamic model, in terms of trajectories and anatomical joints torque, during a squat movement. Once the model had been validated, it would have been used to evaluate the worker's reaction to the shotcrete. The MoCap analysis has been performed using a stereophotogrammetric system for clinical and sports purposes: the BTS SMART-Suite Motion Capture (MoCap) System (by BTS Bioengineering Milan, Italy). A set of 6 infrared cameras has allowed to obtain the three-dimensional coordinates of the 26 markers placed on a subject according to the Extended Davis' protocol. From the markers' trajectories and the anthropometric measurements of the subject, it is possible to obtain the Instantaneous Centers of Rotation (ICRs) position of the anatomical joints. The four digital force platforms, part of the exploited MoCap system, also allow for calculating the torques on the subject's joints starting from the Ground Reaction Force (GRF) measurements they provide.

The kinematics and dynamics validation of the model has been performed on a squat movement, using the MoCap output as a ground reference. The subject has been covered with markers and recorded while performing a full squat motion. The actual Range Of Motion (ROM), as well as the estimated torques for each joint, have been identified. The joint ROM has been fed as input to the dynamic model, and the estimated joint torques have been compared with the simulated ones. Such a comparison is reported in Fig. 5.

The results shown in the figure reveal a good overlap of the knee and ankle torques, while emphasizing a substantial difference on the hip. This discrepancy can be ascribed to two main reasons. Firstly, for sake of simplicity, the dynamic model has been simplified by considering the upper part of the body (trunk, arms, and head) as a single rigid body, whose configuration is the one reported in Fig. 2. Secondly, the model does not take into account the remarkable standard deviation that plagues the ICR position of the hip joint during the motion. This issue will be furtherly addressed in Subsect. 5.1, and a possible solution will be given.

At least in the first instance, the model has been considered a satisfying representation of reality and has been assumed valid. 


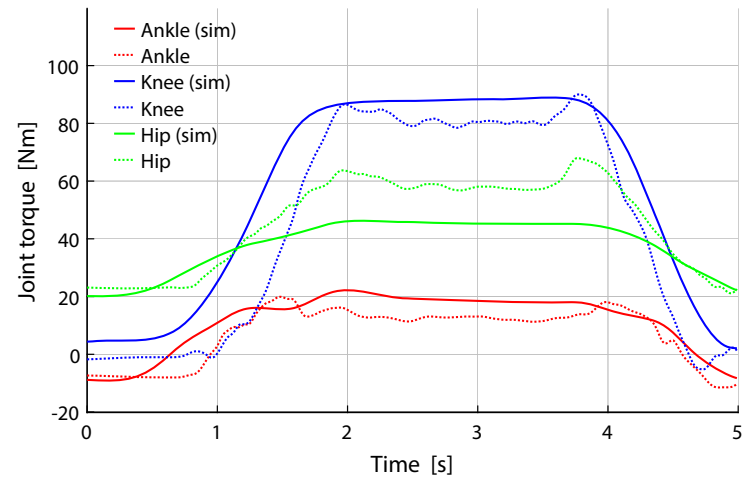

Fig. 5 The comparison between the joint torques from the MoCap system (dashed lines), and the ones simulated by the dynamic model (solid lines)

\subsection{Assessment of the loads on the worker's body}

Once validated, the dynamic model has been used to simulate the working conditions. The total loads on the human joints have been studied in two different simulated conditions: (1) a squat motion to lift a $10 \mathrm{~kg}$ object; (2) a shotcrete projection; both representing the main tasks the workers need to be assisted in.

Firstly, the squat movement has been imposed on the multibody model as the input kinematics. The dynamic model of the worker's body has also been loaded, with an additional $10 \mathrm{~kg}$ to represent the lifting of a heavy object. Such a value has been chosen based on the on-site inspections by observing the workers performing their daily tasks. By using the multibody model, the trend over time of the joint torques necessary to reproduce the imposed motion has been obtained and, later on, compared to the joint torques required to accomplish the same task while wearing the exoskeleton.

Secondly, the shotcrete projection has been simulated using the worker's body posture visible in Fig. 2. This time, the dynamic model has been loaded with all the interaction forces calculated in Sect. 3. Figure 6 shows how the pulsing load, due to the concrete flow and the rhythmic gestures of the workers, is compensated by the worker's joint torques.

\section{Exoskeleton design}

The exoskeleton typology addressed in this work is a fully wearable robotic device designed to assist human
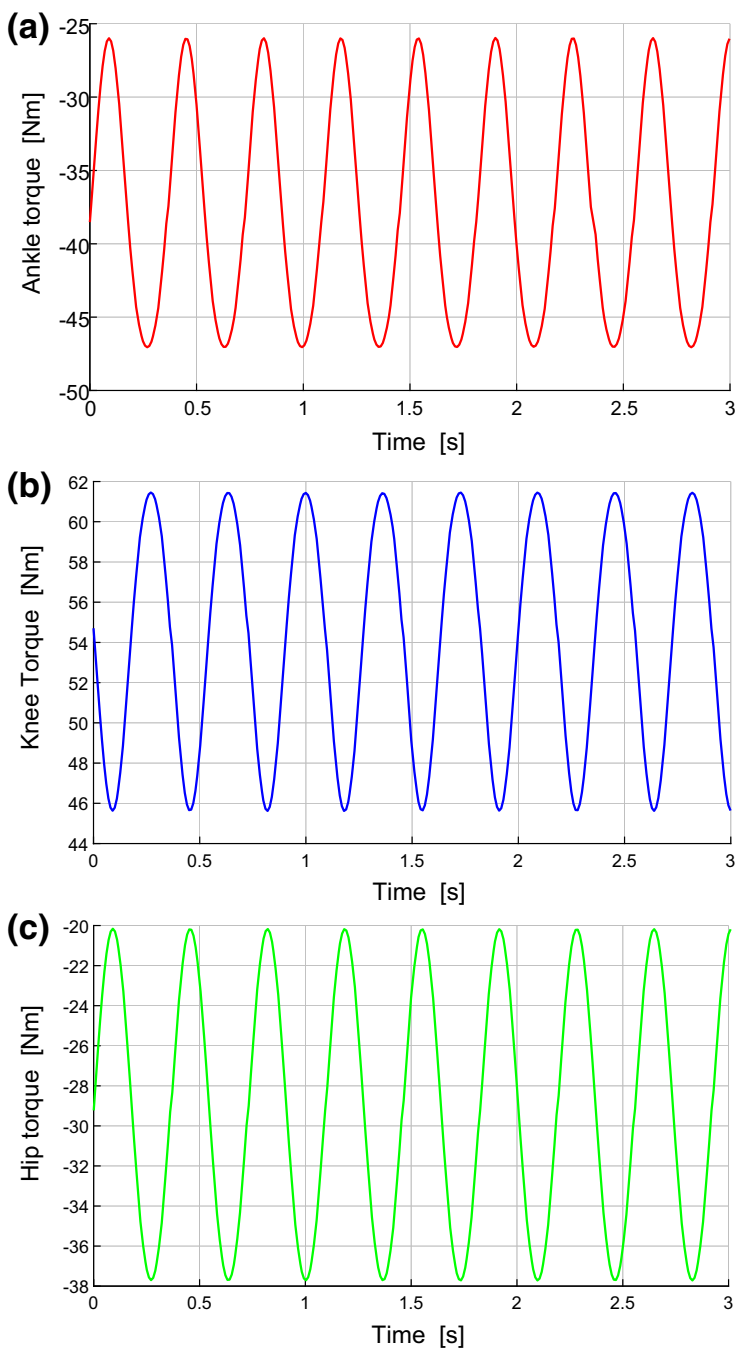

Fig. 6 In $\mathbf{a}, \mathbf{b}$, and $\mathbf{c}$ the torque of the ankle, knee, and hip joint, respectively, during a simulated shotcrete projection

movements and to augment healthy people's performance (e.g., endurance [13, 14] or strength [15]), but also to ensure the wearer to perform some movements correctly [16]. In general, despite the different application fields, all the exoskeletons' common objective is to transfer a controlled amount of power to the user's limbs. Controlling the physical human-robot interaction at the joint level (kinematic coupling) poses several problems in the mechanical design of exoskeletons, which arise mainly from the kinematic complexity and variability of the muscle-skeletal system. The study of the human-robot axes misalignment and the design strategy for the body interface 
covers a vital role in this process, affecting both the efficiency and the safety of the exoskeleton $[17,18]$.

When it comes to transferring the desired torque to a specific wearer's joint, the relative position of the anatomical joint ICRs with respect to the robot joint axis should be fully defined by the geometry and the configuration of the closed kinematic chain created by the connection of the mechanical structure with the user's limb. A correct alignment between the 2 axes is quite challenging to obtain in practice because of the inherent nature of the human musculoskeletal system. However, if this is not deepened, the ICRs will be misaligned, and the torque transfer compromised.

Among all the joints present in the human body, the one which is the most complex to model and reproduce has arisen to be the hip joint $[19,20]$ since the position of this articulation ICR is affected by significant statistical variance in the population. In [21], some data can be found showing the high standard deviation that afflicts hip articulation (more than the other joints), also studied in other researches [22-25]. For these reasons, particular attention has been paid to this joint, whose kinematics has primarily assessed.

The device object of this work has been designed to be used in direct contact with the user to assist his movements and lower the burden on specific skeletal joints (especially the knee and the hip), so it is assumed that interaction between limbs of the human body and the exoskeleton inevitably occur (even for a prolonged time). This interaction needs a human-robot interface whose design is critical because it determines the robot's efficacy, the kinematic compatibility of the device with the human skeletal system, and the level of adaptability to different anthropometries. Failing to deal with such a significant point causes misalignment between the robot and the user's joints; misalignment leads then to the impossibility of adequately controlling the torque transmitted to the user's joints without causing undesired loading forces on other articulations and soft tissues. As a result, this may end up producing an uncomfortable, harmful, or even risky situation for the subject [26].

\subsection{Self-alignment compensating for misalignment}

The MoCap acquisitions have highlighted that even the femur's estimated length (which in reality is a fixed quantity) does not remain constant during the squat: joint ICRs are thus changing their relative positions. In $[27,28]$, the authors formalize such a problem and propose a complete analytical method based on the concept of self-alignment.

Self-alignment means the ability of the mechanism to compensate for the misalignment described in the previous section. This section will describe the alignment of two coupled kinematic chains as they have been $3 \mathrm{D}$ modeled. The first chain represents the human musculoskeletal system. The second chain models the exoskeleton, which should follow the human limbs and, eventually, provide torques along the natural Degrees Of Freedom (DOFs) of the anatomical joints, without affecting the human body chain geometry. From now on, such a robotic device (i.e., an exoskeleton with self-alignment capabilities) will be referred to as a Self-Alignment Mechanism (SAM) $[18,28]$.

As previously mentioned, the joint to which particular attention has to be paid is the hip, which can be mechanically approximated through a spherical joint: a 3-DOF ball-socket joint. This is just an approximation, to which it shall be added that, as already discussed, the ICR position is very likely to change during the motion. Therefore, three additional passive prismatic DOFs have been added to the hip joint along the three principal axes (x, y, and $\mathrm{z}$ ), de facto, modeling it into a 6-DOF joint (Fig. 7). This modeling method allows for the representation of any condition that leads to discrepancy or misalignment. All the errors due to an approximation of the ICR position and the non-perfectly rigid connections between the exoskeleton and the human body are hence compensated.

\subsection{Implementation of the self-aligned system}

In line with the considerations made in the previous section, a mechanism able to follow the motion of the hip has been devised (Fig. 8). The SAM is linked to the human chain in the limb's proximal and distal segments, and the connections are supposed to be rigid. This assumption is borrowed from another study [27]. The idea behind it is that any condition leading to joint axes misalignments - e.g., the apparent variation of the lengths of the anatomical links due to ICR uncertainties, or possible sliding at the interface - can be represented through these additional passive DOFs. 


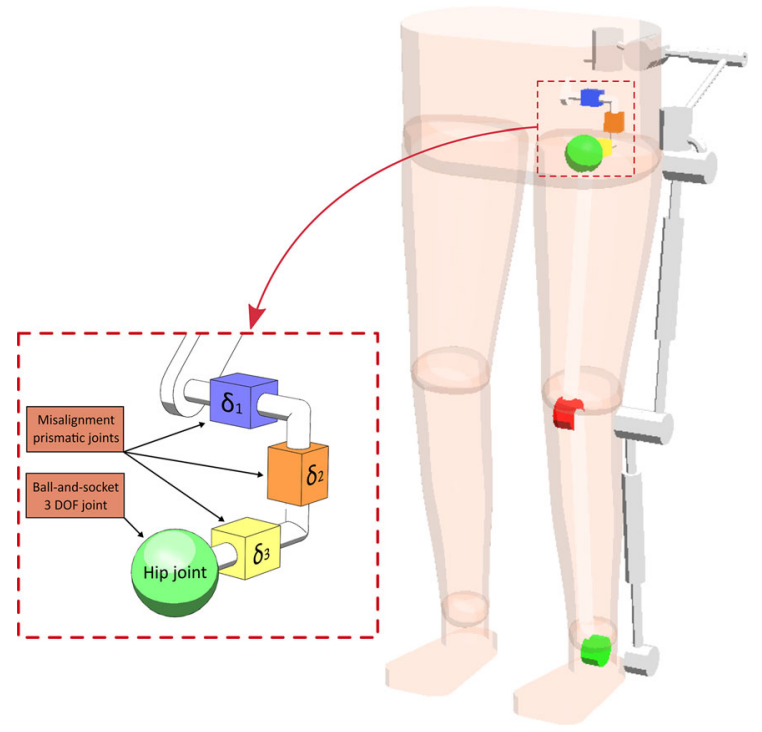

Fig. 7 The modeling of the hip joint: a 3-DOF ball-socket joint as the contact between the head of the femur and the lunate surface of the acetabulum, and 3-DOF prismatic joints $\left(\delta_{1}, \delta_{2}\right.$ and $\delta_{3}$ ) simulating the ICR position uncertainties

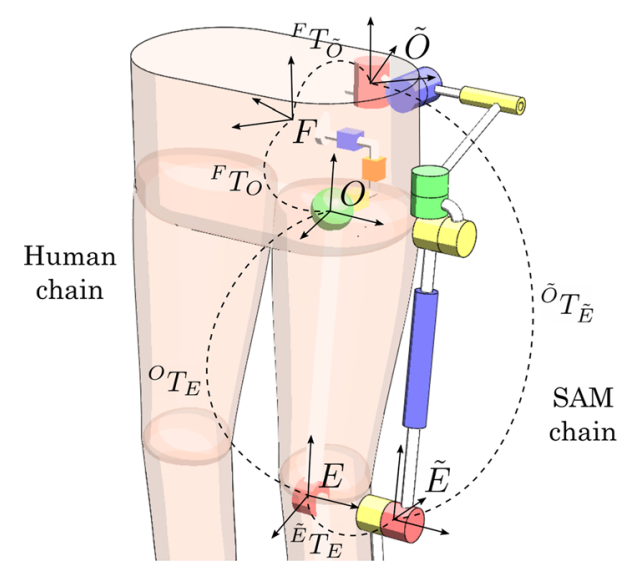

Fig. 8 The kinematic scheme of the proposed mechanism

The 2 serial kinematic chains begin, respectively, from points $O$ (the hip ICR) and $\tilde{O}$ (the center of the reference system integral to the proximal link), and end on points $E$ (the knee ICR) and $\tilde{E}$ (the center of the reference system integral to the distal link). The human joint reference model is described by a set of joint variables $q$ (joints ROMs) and a set of given dimensions $S$ (anthropometric measurements), while the SAM joint variables are $\tilde{q}$. Referring to the Denavit-Hartenberg (D-H) method, the aim of the
SAM design is formally expressed by the following equivalence:

$$
{ }^{F} T_{E}^{(S A M)}(\tilde{q})={ }^{F} T_{E}^{(H)}(q, S),
$$

where $T$ represents a generic homogeneous transformation matrix.

This equation has to consider possible deviations from the reference model to comply with the misalignment issue; otherwise, equality cannot be held. In the following, the additional joints, which represent deviations from the reference model, will be addressed as deviations or misaligning joints, and their variables will be indicated with the $\delta$ symbol. The closure of the complete kinematic chain is expressed as:

$$
\begin{aligned}
{ }^{F} T_{E}^{(S A M)} & ={ }^{F} T_{0}(S, \delta)^{0} T_{E}(q, \delta) \\
& ={ }^{F} T_{E}^{(H)}={ }^{F} T_{\tilde{0}}(\tilde{S})^{\tilde{0}} T_{\tilde{E}}(\tilde{q})^{\tilde{E}} T_{E}(\tilde{S}),
\end{aligned}
$$

where ${ }^{F} T_{O}(S, \delta)$ defines the position of the human chain initial frame, ${ }^{o} T_{E}(q, \delta)$ defines the position of the human chain final frame, ${ }^{F} T_{\tilde{O}}(\tilde{S})$ defines the position of the robot chain initial frame, ${ }^{\sigma} T_{\tilde{E}}(\tilde{q})$ defines the position of the robot chain final frame and determines the robot posture, ${ }^{\tilde{E}} T_{E}(S)$ expresses the connection between the exoskeleton and the human distal link.

The proposed exoskeleton is thus a 6-DOF serial mechanism with its first end-effector on the back, in the lumbar area, and the second one placed on the side of the knee, roughly on the ICR of the natural joint.

Considering Eqs. (11) and (12), the following homogeneous transformation matrix can be defined:

$$
H(q, \tilde{q}, S, \tilde{S}, \delta)={ }^{F} T_{E}^{(S A M)}(\tilde{q}, \tilde{S})-{ }^{F} T_{E}^{(H)}(q, S, \delta)
$$

In case $H$ can be approximated to a null matrix, for any $\delta$ in a range of acceptability (this acceptability range for misalignment will be defined further on), then the proposed SAM can be considered valid because the two chains, that one of the body and the one of the SAM, actually built a closed-loop.

\subsection{Exoskeleton dynamic model}

The exoskeleton dynamic model has been implemented to assess if the joint alignment condition is 
verified once coupled with the human model previously developed. Deviations have been set and used to change the position of the hip joint center arbitrarily. During this dynamic simulation, the hip joint is modeled as a 3-DOF gimbal joint that receives the trend of three angles (ab-adduction, intra-extra rotation, and flex-extension rotation) that together define its motion. The joint's reference system is set to have the three motions as angle variations about the three reference axes. Knee and ankle joints are instead modeled as 1-DOF revolute joints. The combined variation of all these DOFs creates a complex motion that the SAM mechanism has to follow as well as possible.

\subsection{Self-alignment assessment}

Firstly, the coupled multibody model has been used to study the trend of the passive joint stroke as a function of the misalignment. The misalignment is primarily due to the hip ICR position uncertainty, as well as possible deviations to the interface. Such uncertainty has been treated as a three-dimensional error about the position. The nominal position of the hip ICR is defined as the center of an ellipsoidal range of uncertainty, while the deviations $\left(\delta_{1}, \delta_{2}\right.$, and $\left.\delta_{3}\right)$ have been considered the three different semiaxes. According to the literature [21-25], $80 \mathrm{~mm}$ has been chosen as the radius value for the misalignment.

By exploiting the multibody model, an articualte input motion has highlighted that the estimated length of the femur changes during the motion. Obviously, this is not possible and is entirely due to uncertainties about the position of the ICRs during the motion. However, this apparent variation in femur length must be compensated by the exoskeleton. A passive prismatic joint (the blue cylinder on the side of the thigh visible in Fig. 7) has been specially added to adapt the length of the link connecting the (exoskeletal) hip and knee joints accordingly. Once again, having considered a rigid connection between the exoskeleton and the human body has been necessary to determine the passive prismatic joint's maximum stroke. Figure 7 also shows another prismatic joint, which has been added to the shank link as a standard regulation to align the ankle joints.

\section{Assistance to the worker}

The proposed exoskeleton aim is to assist the worker during his construction site duties, mainly focusing on the shotcrete projection and the weight-handling. However, it is crucial, not only for the effective use of the device but also for the safety, that wearer shall not be hindered during any other movement necessary for his duties, i.e., gait and step-over ${ }^{4}$. Another essential requirement was the possibility to wear the exoskeleton, if possible, throughout the shift.

Therefore, a clear design choice has been made develop something that could be used not only to perform the primary task safely, but that could also be worn for a prolonged time: reduce the number of assisted joints and the amount of required assistance (to limit the weight and bulk of the device) in favor of improvement in body posture. Therefore, it has been decided to exploit the same body stance to reduce the loads on the lower limb joints.

Before proceeding with the detailed explanation of this procedure, the fulfillment of the requirement of not obstructing the walk and step-over will be presented. The lower-limb joints' angles during a gait have been derived By exploiting the same MoCap system introduced in Subsect. 4.1: their trends over time are reported in Fig. 9. For what concerns the stepover, the process has been different: the legislation states, in fact, that the tread for a step can vary between 230 and $300 \mathrm{~mm}$, while the riser goes from 160 to 200 $\mathrm{mm}$. Therefore, $200 \mathrm{~mm}$ has been chosen as the reference value for the step riser in the simulation (Fig. 10).

The comparison between the results visible in Fig. 9 and in Fig. 10 shows, for each joint, the ROM boundaries for the exoskeleton assistance. In particular, the knee articulation shall be left free, or unassisted, until almost $65^{\circ}$, as this is needed for the worker to walk without impairments; on the contrary, when the knee joint angle reaches higher values, the assistive system should instead operate, assisting what is likely to be a lifting squat. The assistance limit for the hip joint is instead $60^{\circ}$, meaning that if the hip joint is assisted for smaller angles, the same assistance will interfere with the motion of lifting a leg to climb a

\footnotetext{
${ }^{4}$ Step-over represents the action of bypassing any obstacles: as a generic and representative obstacle a standard-sized step has been chosen
} 


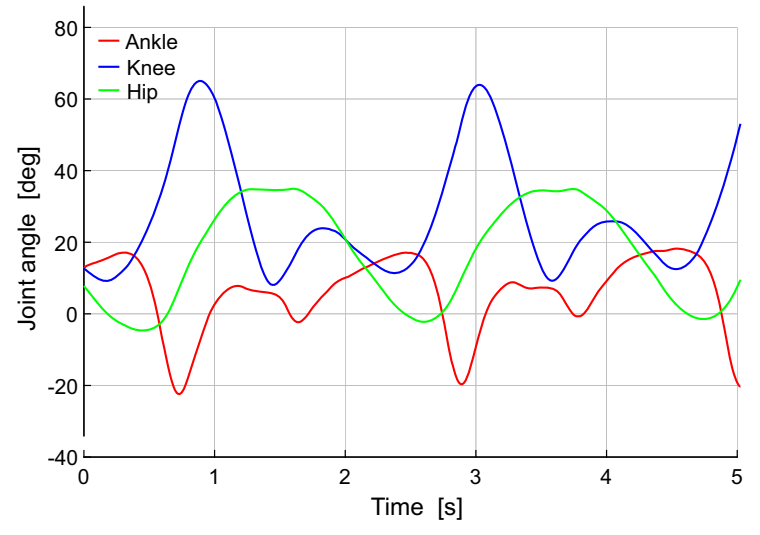

Fig. 9 The trends of the leg joint angles during a standard gait motion

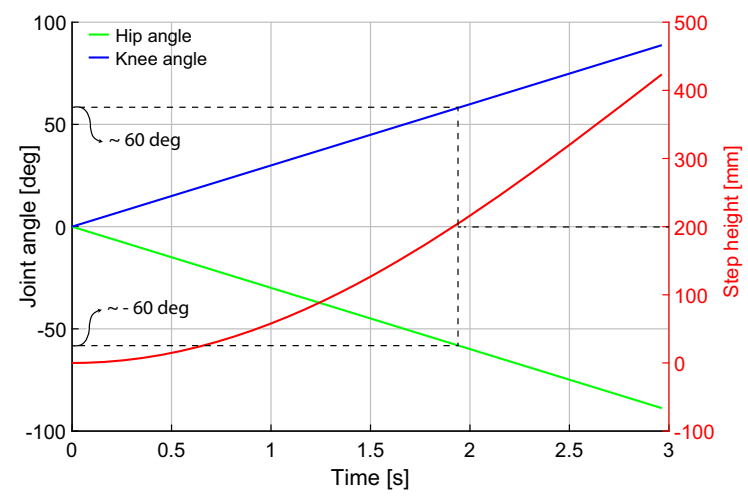

Fig. 10 The simulated trends of the knee (in blue) and hip angles (in green) required to climb over a step. The red line represents the different values for the riser height. The black dashed lines highlight the joint angle for a $200 \mathrm{~mm}$-tall step

step. The ankle should not be assisted in an angular range of $\pm 20^{\circ}$.

\subsection{Improved body posture}

The results of the dynamic shotcrete projection analysis are reported in Fig. 6. These data show that - net of the pulse due to the frequency of worker movement - the hip and ankle joints oppose a negative torque to the forces coming from the hose, while the knee opposes a positive torque. This means that the worker naturally tries to squat forward to counteract the hose force, which, on the contrary, unbalances him backward. From this observation, it has been simulated what would happen if a worker performs the shotcrete projection from a half squat
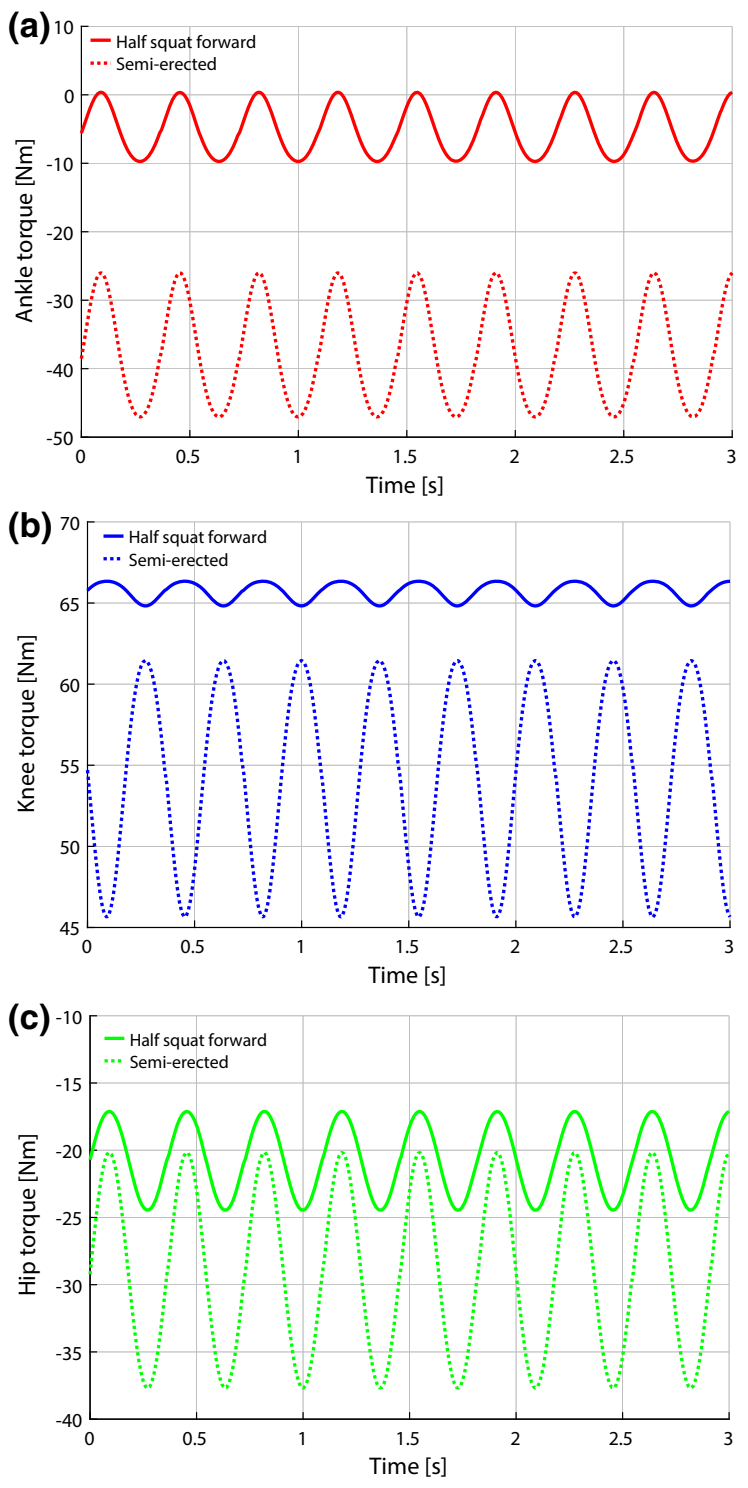

Fig. 11 In a-c, respectively, the comparison between the torque of the ankle, knee, and hip joint during a simulated shotcrete projection in two different body postures

forward position - compliant with the assisted ROM previously identified - instead of the standard semierected body posture. The results of this analysis are reported in Fig. 11, where the simulated joint torques from the semi-erect position are compared with the new one.

By looking at such a comparison, a significant improvement, due to the improved body posture, in the hip and ankle joint conditions - with the average load reduced respectively to about $65 \%$ and $15 \%$ of the 
semi-erected posture - is evident. In contrast, a marked deterioration in the required efforts at the knee joint is visible - with the average load increased by about $20 \%$. The data agree with what practical experience suggests: in fact, the lent forward body helps with its weight on the hip and ankle to counteract the forces from the hose while inevitably loads the knee even more. Moreover, the knee joint appears to be by far more stressed than the others (even in a semierected position), and it is believed that the half squat forward position is not commonly used because it furtherly stresses an already heavily loaded joint.

However, the authors consider that the workers could use this posture to lower their efforts if the exoskeleton could properly assist the knee. While the ankle is almost completely unloaded, the hip joint remains partially loaded. Since the hip — and therefore the lumbar area - is one of the areas most affected by occupational diseases in construction industry workers, the exoskeleton has been developed to provide passive assistance to this joint as well.

\subsection{Knee assistance}

Figure 11 show that the knee is the most loaded joint during the shotcrete projection. Besides, it is wellknown to be the most burdened joints, also during weight-lifting motions. Since both movements need similar assistance - in fact, the knee needs to be supported by a positive couple in both cases ${ }^{5}$ - the best aid system turned out to be a mechanism, similar to a spring, that could assist the wearer proportionally to the required effort, but which could also be locked in position if necessary. The spring could help the worker during the squat; however, if it could also be blocked, it would allow the workers to lean against it and maintain the position without much effort even during the shotcrete projection. The effects of such a system - capable of exerting a force up to $650 \mathrm{~N}$ between the exoskeleton links for the thigh and shank (as shown in Fig. 12) — have been simulated using the multibody model and are reported in Fig. 13.

The use of such an assistance system has shown to lower the torque required to the knee joint by about 60 $\mathrm{Nm}$ when projecting shotcrete; the same system has

\footnotetext{
${ }^{5}$ In simple terms, the knee needs a couple that aims to stretch the limb, mainly counteracting the gravitational force.
}

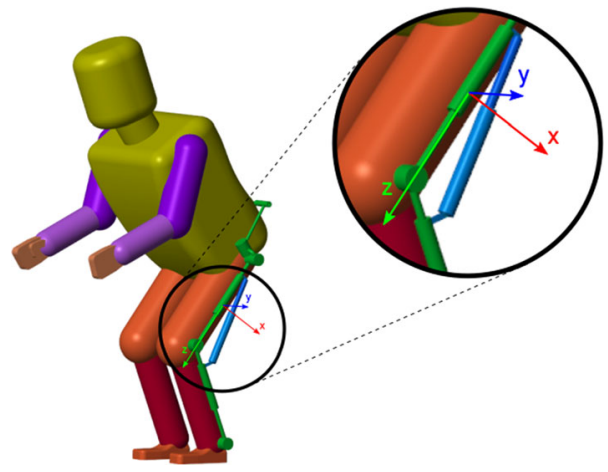

Fig. 12 The dynamic model of the passive knee assist mechanism is reported here in blue. Please note that the configuration of this device is not unique, but rather can be changed according to the level of assistance required
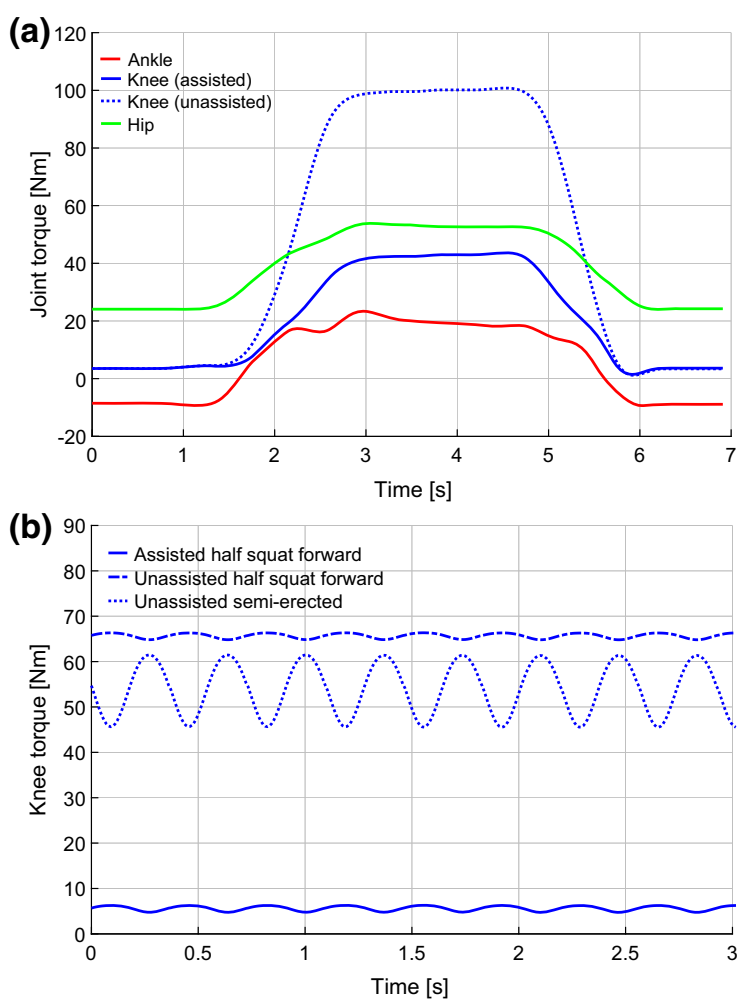

Fig. 13 In a, the comparison between the knee joint torque during a $10 \mathrm{~kg}$-weight-lifting when assisted by the exoskeleton or not: ankle and hip joint are reported just as a reminder; in $\mathbf{b}$, the comparison of the knee joint torque during a shotcrete projection in the 3 different case-studies

proved to reduce the knee joint effort by more than 50 $\mathrm{Nm}$ when a $10 \mathrm{~kg}$ weight has to be lifted. 


\subsection{Hip assistance}

As for the hip, it has been thought to add a physical block - namely, a part of the exoskeleton characterized by a very high stiffness - integral with the thigh link to allow the worker to lean against it during the shotcrete projection, lowering the active burden on the hip joint. The load exerted on the hip during the shotcrete projection is indeed a pulsating torque, which, as reported in Fig. 11, pushes the worker backward (negative-sign torque) and never change its direction. Aiming at avoiding possible movement impairments while performing other activities, the hip angle value which triggers the mechanical stop shall be set to the maximum one identified by the posture of the worker during the projection: which has been determined to be roughly $75^{\circ}$ with respect to the horizontal plane. This is why the mechanical stop has been placed right above the hip joint on the back of the worker: to encourage him to lean on it, releasing the lumbar muscles from the burden, and directly counterpoising its own body mass to the shotcrete effect. Moreover, such a mechanical stop on the hip could also force the worker to assume a correct posture when crouching to lift a heavy object, not allowing to bend the back too much.

\section{Conclusions}

This research's main objective was to design an assistive exoskeleton for construction workers, following the specific requirements imposed by an applicant company. Two crucial tasks have been identified as the most burdening for the workers: shotcrete projection and weight-lifting squat. The same system has to be designed to be used during both of them. Other essential characteristics that have been requested were high wearability and transparency while performing other secondary activities, mainly walking and stepping over an obstacle.

A model-based approach has been fully endorsed to reach this goal. The first step has been the assessment of the scenario the exoskeleton wearer should work within. On the one hand, an on-site measurement campaign has been carried out to evaluate real working conditions. On the other hand, fluid-dynamic calculations (based on these measurements) have been performed, and a dynamic model of the worker's body has been implemented (and validated by MoCap analysis) to estimate the loads that affect the worker. The model has been thus fed with the interaction forces exchanged between the worker and the external environment. Once the effects of the working conditions had been defined, the exoskeleton design has been tackled.

The misalignment issue has been accurately studied, and a possible solution for a self-aligning mechanism has been proposed and developed. Once defined the overall exoskeleton architecture, the device's dynamic model has also been implemented and coupled with the worker's body one. The resulting model has been useful to learn about loads on the worker's joints during shotcrete projection and weight-lifting squat. Moreover, thanks to the proposed model, it has been possible to evaluate the chance to assist the wearer without impairing his other duties (transparency).

The number of actively assisted joints has been reduced to maximize wearability and reduce weight and bulkiness. The use of an improved body posture has proven to be a valid solution to achieve satisfactory assistance levels, minimizing the system's complexity. Besides, it is worth saying that the exoskeleton does not force the worker to the new posture. Instead, its primary aim is to encourage the adoption of a more pronounced leaning-forward stance only for comfort reasons. Promising results have been obtained through the dynamic simulation of shotcrete projection and weight-lifting squat activities: the worker's effort in terms of torque at the anatomical joints - to perform both tasks has been significantly reduced by combining the effects of posture with the direct assistance of the exoskeleton.

Finally, all design parameters have been identified to match all the functional requirements that were the objectives of this work. A design concept of the lower limb passive exoskeleton has been defined. The solution presented here is undoubted designed to perform a particular task. However, applications to other types of work are not to be excluded with appropriate modifications: e.g., assisting workers during the hand-performed cleaning of the streets with the use of pressure washers or while using the jackhammer.

This paper not only aims to show the effectiveness of the design strategy adopted by the authors but also to inspire future research activities towards the 
introduction into the industry of high-tech tools that are both flexible and reliable.

The natural prosecution of this research activity will be the embodiment of the presented concept in an actual prototype. Although at this point of the study, it is still challenging to have a clear idea of all the possible limitations of the device, certainly among them is the fact that the assistance level is closely related to the posture used by the worker, moreover it possible that the exoskeleton use among workers could be hardly accepted. A preliminary estimate of the system's weight identifies it as around $6 \mathrm{~kg}$, symmetrically distributed on the legs. Besides, special fastenings are planned for work shoes to unload part of the weight and the loads on the ground through them. Nevertheless, it is assumed that its use during the entire shift is not entirely painless, mainly because of the additional weight that the worker has to bear. For all these reasons, the device will real-case tested to identify the actual assistance level and evaluate how it influences the metabolic consumption during the workday.

Funding Open access funding provided by Università degli Studi di Firenze within the CRUI-CARE Agreement. This study was carried out in the framework of a research contract funded by Eiffage Infrastructures.

\section{Compliance with ethical standards}

Conflict of interest The authors declare no conflict of interest.

Open Access This article is licensed under a Creative Commons Attribution 4.0 International License, which permits use, sharing, adaptation, distribution and reproduction in any medium or format, as long as you give appropriate credit to the original author(s) and the source, provide a link to the Creative Commons licence, and indicate if changes were made. The images or other third party material in this article are included in the article's Creative Commons licence, unless indicated otherwise in a credit line to the material. If material is not included in the article's Creative Commons licence and your intended use is not permitted by statutory regulation or exceeds the permitted use, you will need to obtain permission directly from the copyright holder. To view a copy of this licence, visit http://creativecommons.org/licenses/by/4.0/.

\section{References}

1. Office of the European Union (2012) Fifth European working conditions survey. Publications office of the European Union, Publication of the European Union 978-92-897-1062-6
2. Goldsheyder D, Weiner SS, Nordin M, Hiebert R (2004) Musculoskeletal symptom survey among cement and concrete workers. Work 23(2):111-121

3. Merlino LA, Rosecrance JC, Anton D, Cook TM (2003) Symptoms of musculoskeletal disorders among apprentice construction workers. Appl Occup Environ Hyg 18(1):57-64

4. Baltrusch S, van Dieën J, Bruijn S, Koopman A, van Bennekom C, Houdijk H (2019) The effect of a passive trunk exoskeleton on metabolic costs during lifting and walking. Ergonomics

5. Kazerooni H, Tung W, Pillai M (2019) Evaluation of trunksupporting exoskeleton. Proc Hum Factors Ergon Soc Ann Meet 63:1080-1083 no. 1. SAGE Publications Sage CA: Los Angeles, CA

6. Poon N, van Engelhoven L, Kazerooni H, Harris C (2019) Evaluation of a trunk supporting exoskeleton for reducing muscle fatigue. In: Proceedings of the human factors and ergonomics society annual meeting. SAGE Publications Sage, CA: Los Angeles, CA. 631 (1): 980-983

7. So B, Cheung H, Liu S, Tang C, Tsoi T, Wu C (2020) The effects of a passive exoskeleton on trunk muscle activity and perceived exertion for experienced auxiliary medical service providers in cardiopulmonary resuscitation chest compression. Intl J Ind Ergon 76:102906

8. Baltrusch S, van Dieën J, Koopman A, Näf M, RodriguezGuerrero C, Babič J, Houdijk H (2020) Spexor passive spinal exoskeleton decreases metabolic cost during symmetric repetitive lifting. Eur JAppl Physiol 120(2):401-412

9. Winter DA (2009) Biomechanics and motor control of human movement. Wiley, Hoboken

10. Drillis R, Contini R, Bluestein M (1969) Body segment parameters. New York University, School of Engineering and Science

11. Zatsiorsky V, Seluyanov V, Chugunova LG (1990) Contemporary problems of biomechanics. Methods of determining mass-inertial characteristics of human body segments, pp 272-291

12. de Leva P (1996) Adjustments to zatsiorsky-seluyanov's segment inertia parameters. J Mech 29(9):1223-1230

13. Walsh CJ, Endo K, Herr H (2007) A quasi-passive leg exoskeleton for load-carrying augmentation. Int J Humanoid Robot 4(03):487-506

14. Pratt JE, Krupp BT, Morse CJ, Collins SH (2004) The roboknee: an exoskeleton for enhancing strength and endurance during walking. In: IEEE international conference on robotics and automation. ICRA'04. 2004. 3: 2430-2435

15. Kazerooni H, Steger R, Huang L (2006) Hybrid control of the berkeley lower extremity exoskeleton (bleex). Int J Robot Res 25(5-6):561-573

16. Perry JC, Rosen J, Burns S (2007) Upper-limb powered exoskeleton design. IEEE/ASME Trans Mech 12(4):408-417

17. Dollar AM, Herr H (2008) Lower extremity exoskeletons and active orthoses: challenges and state-of-the-art. IEEE Trans Robot 24(1):144-158

18. Stienen AH, Hekman EE, Van Der Helm FC, Van Der Kooij H (2009) Self-aligning exoskeleton axes through decoupling of joint rotations and translations. IEEE Trans Robot 25(3):628-633 
19. Bottlang M, Madey S, Steyers C, Marsh J, Brown TD (2000) Assessment of elbow joint kinematics in passive motion by electromagnetic motion tracking. J Orthop Res 18(2):195-202

20. Frisancho AR (1990) Anthropometric standards for the assessment of growth and nutritional status. University of Michigan Press, Ann Arbor

21. Rawal B, Ribeiro R, Malhotra R, Bhatnagar N (2012) Anthropometric measurements to design best-fit femoral stem for the indian population. Indian J Orthop 46(1):46

22. Rubin P, Leyvraz P, Aubaniac J, Argenson J, Esteve P, De Roguin B (1992) The morphology of the proximal femur: a three-dimensional radiographic analysis. J Bone Joint Surg Br 74(1):28-32

23. Husmann O, Rubin PJ, Leyvraz P-F, de Roguin B, Argenson J-N (1997) Three-dimensional morphology of the proximal femur. J Arthroplast 12(4):444-450

24. Mahaisavariya B, Sitthiseripratip K, Tongdee T, Bohez EL, Vander Sloten J, Oris P (2002) Morphological study of the proximal femur: a new method of geometrical assessment using 3-dimensiona reverse engineering. Med Eng Phys 24(9):617-622

25. Noble PC, Alexander JW, Lindahl LJ, Yew DT, Granberry WM, Tullos HS (1988) The anatomic basis of femoral component design. Clin orthop Relat Res 235:148-165

26. Pons JL (2010) Rehabilitation exoskeletal robotics. IEEE Eng Med Biol Mag 29(3):57-63

27. Cempini M, De Rossi SMM, Lenzi T, Vitiello N, Carrozza MC (2013) Self-alignment mechanisms for assistive wearable robots: A kinetostatic compatibility method. IEEE Trans Robot 29(1):236-250

28. Cempini M, Cortese M, Vitiello N (2014) A powered fingerthumb wearable hand exoskeleton with self-aligning joint axes. IEEE/ASME Trans Mech 20(2):705-716

Publisher's Note Springer Nature remains neutral with regard to jurisdictional claims in published maps and institutional affiliations. 